Far Eastern Entomologist

\begin{tabular}{lll}
\hline Number 425: 7-20 & ISSN 1026-051X (print edition) & February 2021 \\
\hline
\end{tabular}

https://doi.org/10.25221/fee.425.2

http://zoobank.org/References/191E3FC3-9FFE-4DA8-B887-E6028469CDEE

\title{
A NEW SPECIES OF THE GENUS CAREBARA WESTWOOD, 1840 (HYMENOPTERA: FORMICIDAE: MYRMECINAE) INHABITS A CAVE IN THAILAND
}

\author{
W. Jaitrong ${ }^{1)}$, P. Pitaktunsakul ${ }^{2 *}$, S. Jantarit ${ }^{3)}$
}

1) Thailand Natural History Museum, National Science Museum, Technopolis, Khlong 5, Khlong Luang, Pathum Thani, 12120 Thailand.E-mail: polyrhachis@ahoo.com

2) Department of General Science, Faculty of Science and Technology, Kanchanaburi Rajabhat University, Kanchanaburi, 71190 Thailand. ${ }^{*}$ Corresponding author, E-mail: ppiyaporn@kru.ac.th

3) Excellence Center for Biodiversity of Peninsular Thailand (CBIPT), Faculty of Science, Prince of Songkla University, Hat Yai, Songkhla, 90110, Thailand. E-mail: fugthong_dajj@ yahoo.com

Summary. A new species Carebara panhai Jaitrong, Pitaktunsakul et Jantarit, sp. n., closely related to C. pygmaea (Emery, 1887), is described from Kanchanaburi Province in Thailand based on the worker caste. The new species is easily distinguished from the closely related species (C. rubra (F. Smith, 1860), C. transversalis (F. Smith, 1860) and C. pygmaea) by the presence of erect hairs on the dorsum of body. New species was found nesting in the twilight zone of a cave. The major and minor workers of C. pygmaea are described and illustrated also.

Key words: ants, Formiciae, taxonomy, new species, cave fauna, Southeast Asia.

В. Ятронг, П. Питактунсакул, С. Янтарит. Новый вид рода Carebara Westwood, 1840 (Hymenoptera: Formicidae: Myrmecinae), обитающий в пещере Таиланда // Дальневосточный энтомолог. 2021. N 425. С. 7-20.

Резюме. Из провинции Канчанабури (Таиланд) по касте рабочих описан Carebara panhai Jaitrong, Pitaktunsakul et Jantarit, sp. n. - вид-двойник муравья C. pygmaea (Emery, 1887). Новый вид отличается от близких видов (C. rubra (F. Smith, 1860), C. transversalis (F. Smith, 1860) и C. pygmaea) характерным опушением дорсальной стороны тела. Гнездо нового вида обнаружено в сумеречной зоне пещеры в Таиланде. Кроме того, приведено иллюстрированное описание крупных и мелких рабочих $C$. pygmaea.

\section{INTRODUCTION}

The ant genus Carebara is distributed worldwide, and is found mainly in tropical and subtropical areas, with 253 described species (Azorsa \& Fisher, 2018). With respect to Carebara pygmaea (Emery, 1887) sensu lato, it has been recorded from a wide range in tropical Asia, including India, Sri Lanka, and various countries of Southeast Asia (Wheeler, 
1909; Pfeiffer et al., 2011; Jaitrong et al., 2016; Antweb, 2020; Herwina et al., 2020; Khachonpisitsak et al., 2020). However, C. pygmaea was transferred to the genus Carebara Westwood, 1840 by Fischer et al. (2014). We have examined rich material of C. pygmaea sensu lato collected from Thailand and Laos and compared them with high-resolution images of syntypes of $C$. pygmaea from Ternate Island, Indonesia. After careful study, we have reached the conclusion that all specimens examined belong to C. pygmaea, except a colony collected in a cave from Sahakhon Nikhom Village, Thong Pha Phum District, Kanchanaburi Province that differ from the syntypes of C. pygmaea in some characters and nesting habits.

In the present paper, we describe this unknown species as new to science based on the worker castes (major and minor workers). Carebara pygmaea is also redescribed. Ecological notes and distribution of both species are discussed.

\section{MATERIAL AND METHODS}

The holotype and paratypes were pin-mounted dry specimens collected in the Kanchanaburi Province of Thailand. The type material of the new species was compared with high-resolution images of syntypes of the closely related species of the Carebara pygmaea species group available on Antweb (2019).

Most morphological observations were made with a ZEISS Discovery V12 stereoscope. Multi-focused montage images were produced using NIS-Elements-D-[Sequence $6 *$-Focused] from a series of source images taken by a Nikon Digital Sight-Ril camera attached to a Nikon AZ100M stereoscope. Type specimens of each species were measured for the following parts using a micrometer (accurate to $0.01 \mathrm{~mm}$ ).

The abbreviations used for the measurements and indices are as follows: TL - Total length roughly measured from anterior margin of head (including mandible) to tip of metasoma in stretched specimens, excluding sting; HL - Head length: maximum length of cranium in full-face view, measured from transverse line spanning the anteriormost points of clypeus to that of posteriormost points of cranium; HW - Head width: maximum width of cranium in full-face view (excluding eyes); SL - Scape length: maximum length of antennal scape excluding basal condylar bulb; ML - Mesosomal length: maximum diagonal length of mesosoma in lateral view, measured from posterodorsal border of pronotal flange to posterior basal angle of metapleuron; MW - Mesosomal width: maximum width of promesonotum in dorsal view; PL - Petiolar length: maximum length of petiole in lateral view (excluding helcium); PH - Petiolar height: maximum height of petiole in lateral view (including subpetiolar process); DPW - Dorsal Petiole Width. The maximum width of the petiole in dorsal view; CI - Cephalic index: HW/HL $\times 100 ;$ SI - Scape index: SL/HW $\times 100 ;$ PI1 - Petiolar index 1: $\mathrm{PL} / \mathrm{PH} \times 100 ; \mathbf{P I} 2-$ Petiolar index 2: DPW/PL $\times 100$.

Abbreviations of the type depositories are as follows: MHNG - Muséum d'histoire naturelle, Geneva, Switzerland; SKYC - Seiki Yamane Collection, Japan; THNHM - Natural History Museum of the National Science Museum, Thailand.

\section{TAXONOMY}

\section{Genus Carebara Westwood, 1840 \\ Carebara pygmaea species group}

DESCRIPTION. Dimorphism in worker caste. Major worker. Head larger than thorax; frons with longitudinal striation; frontal carinae with 3 pairs of erect hairs; mandible relatively 
short, subtriangular, masticatory margin with 5 teeth (including large apical tooth); antenna 11-segmented with 2-segmented club; antennal scape thin (clavate, its apical portion narrower than or broad as long as antennal segment $\mathrm{X}$ ); promesonotum in profile view distinctly convex to form a dome; promesonotal suture indistinct; metanotal groove distinct; propodeum distinctly lower than promesonotum; propodeal spine short, usually shorter than its base; petiole, in profile, with long anterior pedicel; postpetiole clearly shorter than petiole; Gastral tergite I clearly large than remaining segments. Minor worker. Head small, smooth and shiny; mandible relatively short, subtriangular, masticatory margin with 5 teeth (including large apical tooth); eye very small, present as a dot; antenna 11-segmented with 2-segmented club; antennal scape thin (clavate, its apical portion narrower than or almost as broad as antennal segments $\mathrm{X}$ ); promesonotum in profile view convex, smooth and shiny; promesonotal suture absent; metanotal groove distinct; mesopleuron and propodeum largely punctate; propodeal spine absent; petiole, in profile, with long anterior pedicel; postpetiole clearly shorter than petiole; Gastral tergite I clearly large than remaining segments. Dealate geny. Head smaller than mesosoma; frons with longitudinal striation; eye large and convex; three ocelli present on vertex; pronotum in profile placed in vertical, subrectangular, shorter than mesoscutum; masoscutum large, located higher than pronotum, subtrapezoidal, its anterior margin strongly convex; parapsidal line long; propodeal spine short; petiole, postpetiole and gaster as in major workers.

COMPOSITION AND DISTRIBUTION OF THE SPECIES GROUP. Carebara pygmaeus (Emery, 1887), C. rubra (Smith, 1860), C. transversalis (Smith, 1860), and C. panhai sp. n. are known from Sri Lanka, Laos, Thailand, Malaysia, Indonesia, and the Philippines.

Carebara panhai Jaitrong, Pitaktunsakul et Jantarit, sp. n.

http://zoobank.org/NomenclaturalActs/76B1D229-0B0D-4A99-92E6-5A7647283212

Figs 1,2

TYPE MATERIAL. Holotype - major worker (THNHM-I-14191), Thailand: Kanchanaburi Province, Thong Pha Phum District, Sahakhon Nikhom Community Forest, $14.76255556^{\circ} \mathrm{N}, 98.80966667^{\circ} \mathrm{E}, 3 \mathrm{~m}$ from a cave entrance, 27.XI 2019, W. Jaitrong leg. (THNHM). Paratypes: same data as holotype, 4 major workers (THNHM-I-14192, THNHM-I19032 to THNHM-I-19034; SKYC, THNHM) and 60 minor workers (THNHM-I-14193, THNHM-I-19035 to THNHM-I-19093; SKYC, THNHM)

MEASUREMENTS. Holotype: TL 3.80, HL 1.10, HW 0.96, SL 0.56, ML 0.86, MW 0.50, PL 0.36, PH 0.33, DPW 0.23, CI 83, SI 61, PI1 122, PI2 60. Major worker (four paratypes): TL 3.603 .80 , HL 1.09-1.10, HW 0.89-0.96, SL $0.53-0.56$, ML $0.79-0.86$, MW $0.46-0.50$, PL $0.33-0.36$, PH 0.30-0.33, DPW 0.20-0.23, CI 82-83, SI 59-61, PI1 110-122, PI2 55-60. Minor worker (ten paratypes): TL 2.15-2.18, HL 0.53-0.56, HW 0.46, SL 0.46 , ML 0.53-0.56, MW 0.25-0.26, PL 0.26-0.30, PH 0.17, DPW 0.10, CI 82-88, SI 100, PI1 $150-180$, PI2 33-38.

DESCRIPTION. Major worker (Fig. 1A-C). Head, in full-face view, subrectangular, longer than broad, almost parallel sides, posterior cephalic margin shallowly concave medially, and posterolateral corner roundly convex; cephalic median furrow developed, extending anteriad to posterior one third of head length; in dorsal view, posterior one third of head with irregular transvers striation, anterior two third of head with longitudinal striation; lateral (area below eye) face of head somewhat smooth and shiny; dorsum of head covered entirely with sparse erect hairs mixed with dense short decumbent hairs; mandible short, subtriangular, smooth and shiny; mandible with sparse decumbent hairs; anterior clypeal margin 
weakly convex; median portion of clypeus smooth and shiny; frontal lobe poorly developed, only partly covering antennal socket; frontal carina inconspicuous; eye relatively small, with 12-13 ommatidia, located at anterior one third of head length laterally; antenna 11-segmented with 2-segmented club; antennal scape thin (clavate, its apical portion almost as long as antennal segments X) and short, when laid backward reaching posterior one third of head length; scape somewhat smooth and shiny, covered with dense decumbent hairs.

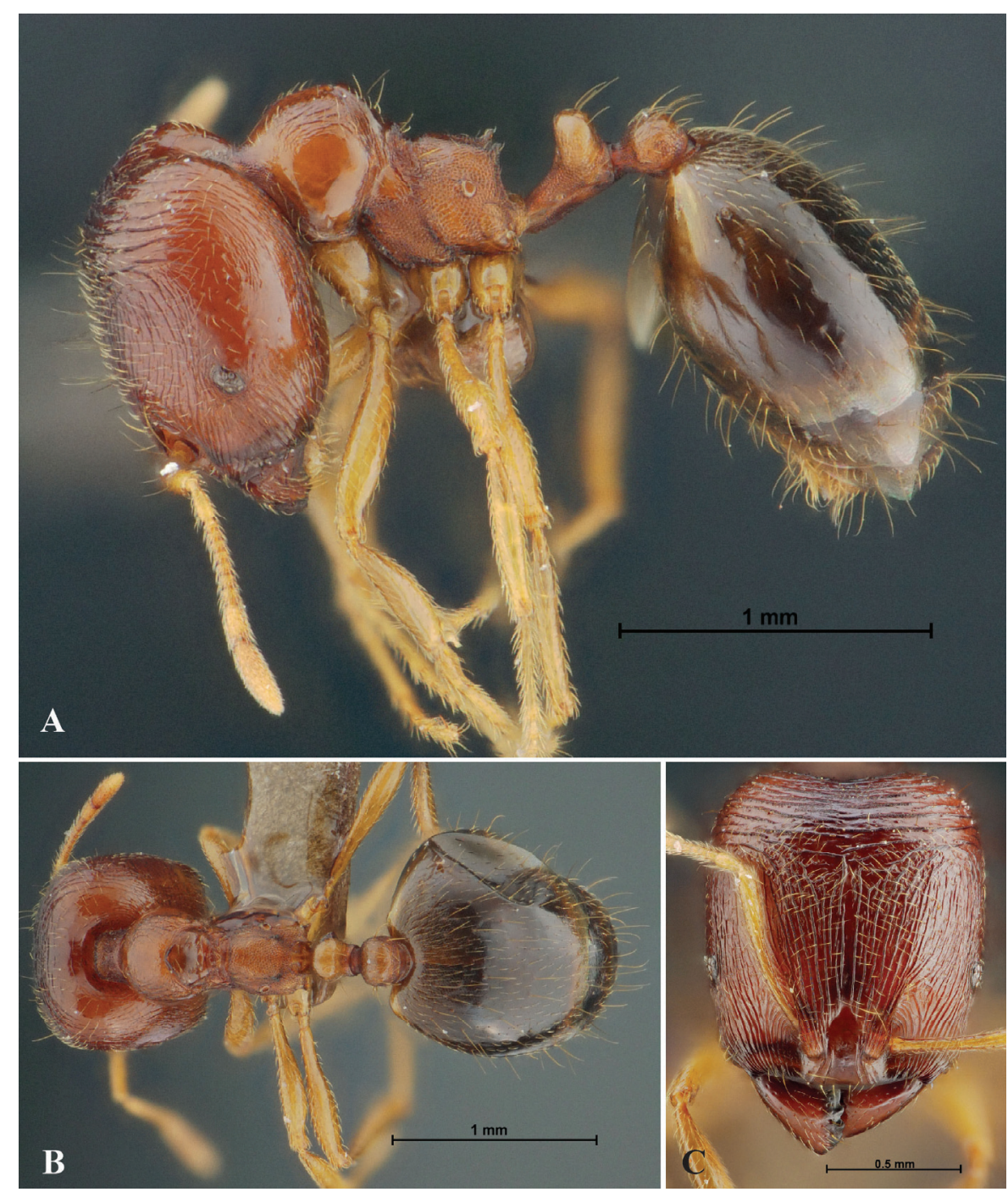

Fig. 1. Carebara panhai sp. n., holotype major worker (THNHM-I-14191). A - body in profile; $\mathrm{B}$ - dorsal view of body; $\mathrm{C}$ - head in full-face view. 
Mesosoma smaller than head and gaster; promesonotum strongly convex to form a dome, mesosonotum slope to metanotal groove, with sparse erect hairs mixed with dense short decumbent hairs; promesonotal suture present as carina dorsally; pronotal dorsum wrinkled but slightly shiny, lateral face of pronotum smooth and shiny; mesonotum smooth and shiny; mesopleuron punctate, clearly demarcated from metapleuron by deep groove; metanotal groove distinct; propodeal spine, in profile, relatively short and subtriangular, pointed posterolaterally; in profile view, propodeum distinctly lower than promesonotum, its dorsal outline almost straight; propodeum entirely punctate; entire mesosoma without erect hairs; legs entirely with numerous short decumbent hairs, smooth and shiny.

Petiole, in profile, with long anterior pedicel, punctate and without hairs; petiolar node, in profile, relatively short, shorter than high, its lateral face micropunctate, while dorsal face smooth and superficially shagreened with smooth and shiny interspaces, with 4-5 erect hairs; postpetiole clearly shorter than broad, its dorsal outline weakly convex, and entirely finely punctate; in dorsal view, postpetiole rounded; postpetiole with 4-6 erect hairs.

Gaster larger than head and elliptical; gastral tergite I smooth and shiny, and covered with dense erect hairs mixed with dense short decumbent hairs; anterior margin of gastral tergite I feebly concave.

Head, mesosoma and waist (petiole and pospetiole) reddish brown; gaster dark brown; antenna and legs yellowish brown.

Minor worker (Fig. 2A-C). Head, in full-face view, longer than broad, lateral margin weakly convex and posterior cephalic margin almost straight; head entirely smooth and shiny; dorsum of head entirely covered with dense short decumbent hairs, a pair of erect hairs on frons, and a pair of erect hairs on frontal carinae; mandible short, subtriangular, smooth and shiny; clypeus entirely smooth and shiny, its anterior clypeal margin roundly convex; frontal lobe poorly developed, only partly covering antennal socket; frontal carina inconspicuous, reaching level of eye; eye relatively small, with one ommatidium, located at anterior $1 / 4$ of head length laterally; antenna 11 -segmented with 2 -segmented club; antennal scape thin (clavate, its apical portion almost as broad as antennal segment $\mathrm{X}$ ), when laid backward extending slightly beyond posterolateral corner of head; scape somewhat smooth and shiny, with dense decumbent hairs.

Mesosoma narrower than head and gaster; promesonotum strongly convex to form a dome, mesonotum slope to metanotal groove, with dense short decumbent hairs; promesonotal suture absent, promesonotum entirely smooth and shiny; mesopleuron finely punctate, clearly demarcated from metapleuron by deep groove; metanotal groove distinct; metapleuron not demarcated with lateral face of propodeum, largely smooth and shiny; propodeal spine absent, in profile view, propodeum distinctly lower than promesonotum, its dorsal outline weakly convex; propodeal junction convex; declivity of propodeum shallowly concave, marginated laterally by thin rim; dorsum of propodeum smooth and shiny, its lateral face punctate posteriorly; entire mesosoma without erect hairs; legs entirely with numerous short decumbent hairs, smooth and shiny.

Petiole, in profile, with long anterior pedicel, micropunctate and without hairs; petiolar node, in profile, shorter than high, convex dorsal outline, smooth and shiny, with $2-4$ erect hairs; postpetiole in profile view, almost flat, with weakly convex dorsal outline, smooth and shiny, with 2-4 erect hairs.

Gaster larger than head; gastral tergite I smooth and shiny, and covered with dense short decumbent hairs mixed with sparse erect hairs; anterior margin of gastral tergite I feebly concave. Body yellowish brown.

DIAGNOSIS. New species is most similar to C. pygmaea. Both species share the following characteristics of major workers: frons with longitudinal striation, while vertex with transverse 
striation; frontal carinae with 3 pairs of erect hairs; masticatory margin with 5 teeth (including large apical tooth); antenna 11-segmented with 2-segmented club; promesonotum in profile view distinctly convex to form a dome; promesonotal suture indistinct; propodeal spine short, usually shorter than its base. However, C. panhai can be easily distinguished from C. pygmaea by the following characteristics: 1) in major workers, frons, vertex dorsa of petiole and postpetiole and first gastral tergite with dense erect hairs (without erect hairs in C. pygmaea); 2) lateral face of pronotum largely smooth and shiny (punctate and reticulate in C. pygmaea); propodeum in minor workers largely smooth and shiny (entirely punctate in C. pygmaea).

HABITAT. The type series was collected from a cave at twilight zone about $3 \mathrm{~m}$ from the entrance.

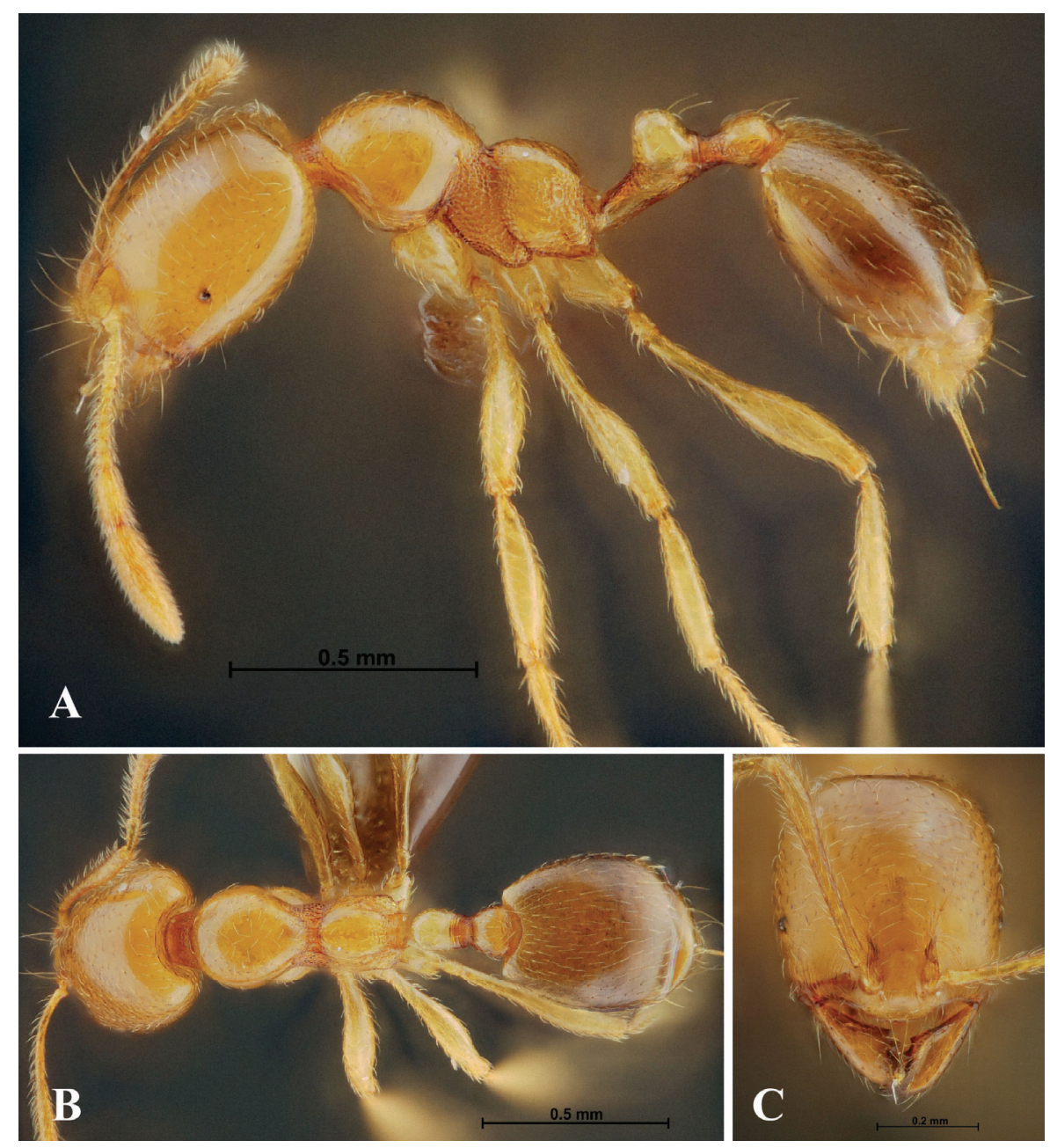

Fig. 2. Carebara panhai sp. n., paratype minor worker (THNHM-I-14193). A - body in profile; $\mathrm{B}$ - dorsal view of body; $\mathrm{C}$ - head in full-face view. 
DISTRIBUTION. Thailand (Kanchanaburi Province).

ETYMOLOGY. The specific name is dedicated to Professor Somsak Panha (the Director of the Higher Education Commission and Centre of Excellence on Biodiversity, Chulalongkorn University), a famous taxonomist in Thailand, who is dedicated to karst and cave biodiversity and conservation in the country and kindly supported us in field surveys.

\section{Carebara pygmaea (Emery, 1887)}

Figs 3, 4

Pheidologeton pygmaeus Emery, 1887: 465 (type locality: Indonesia, Ternate Island); Ettershank, 1966: 119.

Pheidologeton (Aneleus) pygmaeus: Emery, 1900: 327.

Aneleus pygmaeus: Emery, 1924: 214.

Carebara pygmaeus: Fischer et al., 2014: 72; Khachonpisitsak et al., 2020: 82.

Pheidologeton pygmaeus var. albipes Emery, 1893: 266 (type locality: the Philippines); Ettershank, 1966: 118. Junior synonym of pygmeae (Fischer et al., 2014: 72).

Aneleus pygmaeus var. albipes: Emery, 1924: 214

Pheidologeton (Aneleus) pygmaeus var. bugnioni Forel, 1915: 28 (type locality: Sri

Lanka); Ettershank, 1966: 118. Junior synonym of pygmeae (Fischer et al., 2014: 72).

Aneleus pygmaeus var. bugnioni: Emery, 1924: 214.

Pheidologeton (Aneleus) pygmaeus var. simalurensis Forel, 1915: 27 (type locality:

Indonesia, Sumatra). Junior synonym of pygmeae (Fischer et al., 2014: 72).

Aneleus pygmaeus var. simalurensis: Emery, 1924: 214.

Oligomyrmex simalurensis: Ettershank, 1966: 124.

Carebara pygmaeus var. simalurensis: Fernández, 2004: 235.

MATERIAL EXAMINED. Northern Thailand: Chiang Rai Prov., Mae Fa Luang Dist., Mae Fa Luang Subdist., Doi Tung, 589 m a.s.l., 20 $16^{\prime} 19^{\prime \prime}$ N, 99 $49^{\prime} 19^{\prime \prime}$ E, 22.X.2018, W. Jaitrong leg., WJT221018-43 (THNHM). Western Thailand: Tak Prov., Umphang Dist., Thung Yai Naresuan East W.S., Thung Na Noi Station, 20.II.2015, W. Jaitrong leg., TH15WJT-411 (THNHM); Kanchanaburi Prov., Thong Pha Phum Dist., Maeklong Watershed Research Station, 29.XI.2003, Sk. Yamane leg., TH03-SKY-145 (SKYC, THNHM). Eastern Thailand: Chonburi Prov., Khao Ang Reu Nai W.S., Ang Phaknam Station, dry evergreen forest, 3.IV.2004, W. Jaitrong leg., WJT04-E006 (THNHM); Rayong Prov., Khao Ang Reu Nai W.S., Siraman Station, dry evergreen forest, 5.IV.2004, W. Jaitrong leg., WJT04-E028 (THNHM); Chachoengsao Prov., Thatha Keab Dist., Khao Ang Reu Nai W.S., Bo Thong Waterfall, 27.IV.2003, W. Jaitrong leg., WJT03-TH-5 (THNHM); Chanthaburi Prov., Pheao N.P., Troknong Waterfall, 23.XI.2003, W. Jaitrong leg., TH03-WJT-580 (THNHM); same locality, date and collector, TH03-WJT-563 (THNHM); Chanthaburi Prov., Soi Dao Dist., Khao Soi Dai W.S., Head Quarter, 14.IV.2008, W. Jaitrong leg., WJT08-E062 (THNHM); Chanthaburi Prov., Pong Nam Ron Dist., Khlong Tab Mak Waterfall, 18.V.2008, W. Jaitrong leg., WJT08-E181 (THNHM). Southern Thailand: Surat Thani Prov., Tha Chang Dist., Tha Chang Subdist., evergreen forest, 27.II.2020, W. Jaitrong leg., TH20-WJT21 (THNHM); Nakhon Si Thammarat Prov., Sichon Dist., Khao Nan N.P., Yod Nam Waterfall, 239 m a.s.l., 11.XII.2007, W. Jaitrong leg., WJT07-TH1886 (THNHM); Trang Prov., Nayong Dist., Khao Chong B.G., 23.II.2020, W. Jaitrong leg., TH20-WJT-02 (THNHM).

MEASUREMENTS. Major worker $(\mathrm{n}=10)$ : TL 4.13-4.16, HL 1.19-1.22, HW 1.021.06, SL 0.53-0.56, ML 0.89-0.96, MW 0.59-0.63, PL 0.33-0.35, PH 0.26-0.30, DPW 0.26 , CI 86, SI 52-53, PI1 117-125, PI2 76-80. 
Minor worker $(\mathrm{n}=10)$ : TL 2.02-2.08, HL 0.46-0.50, HW 0.46-0.48, SL 0.36-0.40, ML 0.50-0.53, MW 0.23-0.26, PL 0.20-0.23, PH 0.17, DPW 0.10, CI 93-100, SI 79-86, PI1 120-140, PI2 43-50.

Major worker (Fig. 3A-C). Head, in full-face view, almost as long as broad, lateral margin weakly convex, posterior cephalic margin shallowly concave, and posterolateral corner roundly convex; cephalic median furrow indistinct; in dorsal view, posterior one fourth of head with fine transverse striation, anterior three fourth of head with fine longitudinal striation; lateral (area below eye) face of head punctate (in some specimens slightly shiny); head

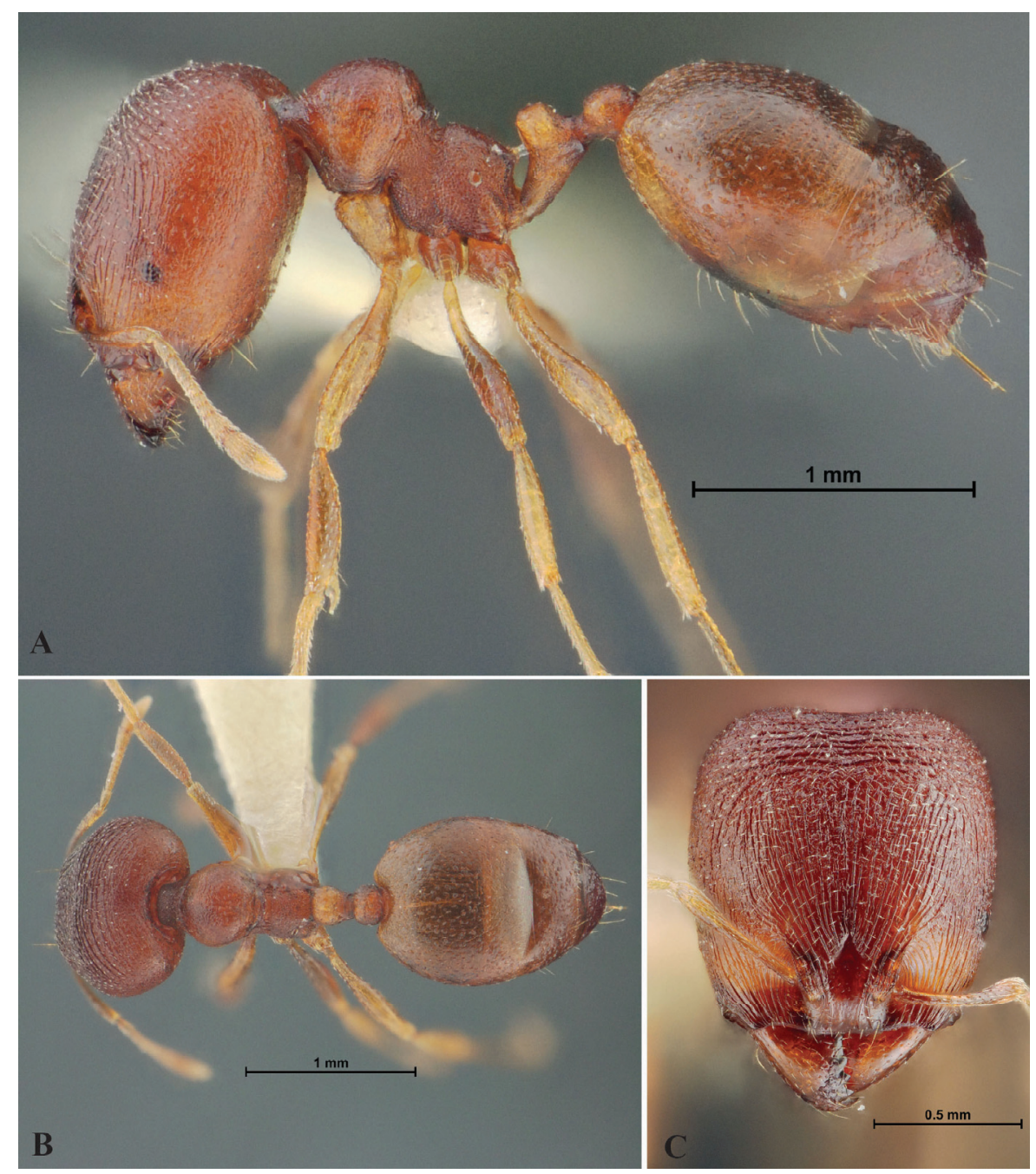

Fig. 3. Carebara pygmaea, major worker (THNHM-I2013-05969 [= TH03-SK-145]). A body in profile; $\mathrm{B}$ - dorsal view of body; $\mathrm{C}$ - head in full-face view. 
with three pairs of erect hairs on frontal carinae and dense very short hairs over surface, vertex and frons without erect hairs; mandible short, subtriangular, smooth and shiny; mandible with sparse decumbent hairs; anterior clypeal margin weakly convex; median portion of clypeus smooth and shiny; frontal lobe poorly developed, only partly covering antennal socket; frontal carina conspicuous, reaching level of anterior margin of eye; eye relatively small, with 12-13 ommatidia, located at anterior one third of head length laterally; antenna 11-segmented with 2-segmented club; antennal scape thin (clavate, its apical portion almost as long as antennal segments X) and short, when laid backward reaching posterior one third of head length; scape somewhat smooth and shiny, covered with dense decumbent hairs.

Mesosoma smaller than head and gaster; promesonotum strongly convex to form a dome, mesosonotum slope to metanotal groove; promesonotal suture indistinct; pronotum punctate; mesonotum punctate; mesopleuron punctate, clearly demarcated from metapleuron by deep groove; metanotal groove distinct; propodeal spine, in profile, short and subtriangular, shorter than its base; in profile view, propodeum distinctly lower than promesonotum, its dorsal outline almost straight; metapleuron and propodeum entirely punctate; mesosoma without hairs; legs smooth and shiny without hairs; punctation on pronotum weaker than on mesopleuron and propodeum.

Petiole, in profile, with long anterior pedicel, punctate and without hairs; petiolar node, in profile, shorter than high, smooth and shiny, without hairs; postpetiole round, its dorsal outline convex, punctate, without hairs.

Gaster slightly smaller than head and elliptical; gastral tergite I superficially reticulate with smooth and shiny interspaces, without hairs; anterior margin of gastral tergite I concave.

Body reddish brown to dark brown; gaster dark brown; antenna and legs reddish brown.

Minor worker (Fig. 4A-C). Head, in full-face view, as long broad, lateral margin weakly convex and posterior cephalic margin weakly concave; head entirely smooth and shiny; dorsum of head entirely covered with dense short decumbent hairs, and a pair of erect hairs on frontal carinae; mandible short, subtriangular, smooth and shiny; clypeus entirely smooth and shiny, its anterior clypeal margin roundly convex; frontal lobe poorly developed, only partly covering antennal socket; frontal carina inconspicuous; eye relatively small, with one ommatidium, located at anterior one fourth of head length laterally; antenna 11-segmented with 2-segmented club; antennal scape thin (clavate, its apical portion almost as broad as antennal segments X), when laid backward almost reaching posterolateral corner of head; scape somewhat smooth and shiny, without hairs.

Promesonotum strongly convex to form a dome, mesonotum slope to metanotal groove, with dense short decumbent hairs; promesonotal suture absent, promesonotum entirely smooth and shiny; mesopleuron finely punctate, clearly demarcated from metapleuron by deep groove; metanotal groove distinct; metapleuron punctate, not demarcated with lateral face of propodeum; propodeal spine absent, in profile view, propodeum distinctly lower than promesonotum, its dorsal outline weakly convex; propodeal junction convex; declivity of propodeum shallowly concave, marginated laterally by thin rim; dorsum of propodeum smooth and shiny, its lateral face punctate; mesosoma without erect hairs; legs smooth and shiny, without hairs.

Petiole, in profile, with long anterior pedicel, micropunctate and without hairs; petiolar node, in profile, shorter than high, convex dorsal outline, smooth and shiny, without hairs; postpetiole in profile view, round, convex dorsal outline, smooth and shiny, without hairs.

Gastral tergite I smooth and shiny, and covered with dense short decumbent hairs but without erect hairs; anterior margin of gastral tergite I concave. Body reddish brown to dark brown. 
DIAGNOSIS. Carebara pygmaea is most similar to Carebara rubra (Smith, 1860) and Carebara transversalis (Smith, 1860) in lacking erect hairs on head, mesosoma, and gastral tergite I (except 3 pairs on frontal carinae). However, this species can be separated from the latter two by: the shot propodeal spine, shorter than its base or almost absent (relatively longer than their base in the letter two) and punctate lateral face of pronotum, petiolar node, and postpetiole (slightly smooth and shiny in the latter two).
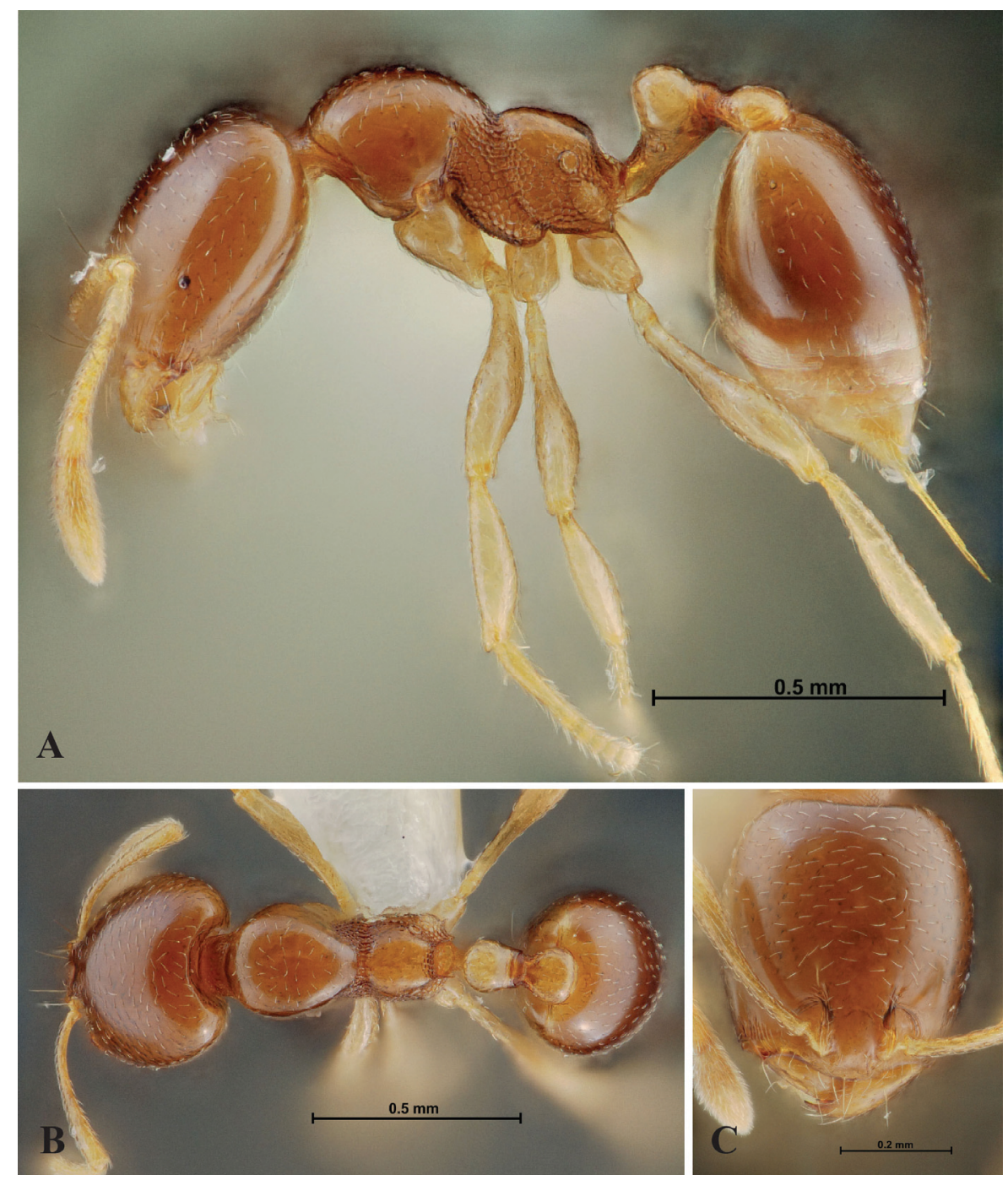

Fig. 4. Carebara pygmaea, minor worker (THNHM-I2013-05970 [= TH03-SK-145]). A body in profile; $\mathrm{B}$ - dorsal view of body; $\mathrm{C}$ - head in full-face view. 
HABITAT. This species inhabits both disturbed and primary forests and from lowlands to highlands (100-1000 m a.s.l.). Most colonies were collected from dead logs on forest floor. One colony (TH20-WJT-21) was collected from the dead part (stem) of a living tree.

DISTRIBUTION. Sri Lanka, Laos (Vientiane Province), Thailand (Chiang Rai, Tak, Kanchanaburi, Chonburi, Rayong, Chachoengsao, Chanthaburi, Surat Thani, Nakhon Si Thammarat, and Trang Provinces), Malaysia (Borneo), Indonesia (Sulawesi, Sumatra, and Ternate), and the Philippines.

\section{DISCUSSION}

Carebara pygmaea appears to be distributed throughout Thailand. It inhabits both primary and secondary forests where most colonies are collected from rotting logs on the forest floor. The new species is sympatric with $C$. pygmaea at least in western Thailand, Kanchanaburi Province, Thong Pha Phum District. Carebara panhai sp. n. is easily distinguished from its related species C. pygmaea, C. rubra, and C. transversalis by the presence of erect hairs on dorsum of body (head, mesosoma, petiole, postpetiole, and first gastral tergite). Regarding to its ecology, it has long been known that most Carebara species nest in the soil, the leaf litter or in termite mounds (Antwiki, 2020). However, the type series of this new species was found nesting in the twilight zone of a cave in a deep hole on a cave wall, about $3 \mathrm{~m}$ from the entrance and $2 \mathrm{~m}$ from the ground. The entrance of the nest is small about $3.2 \times 3 \mathrm{~cm}$ (Fig. 5). This is the first report of the genus living in cave environment indicating the ecological niche of the Carebara is not limited only hypogaeic habitat. During the observation, workers (mainly minor) were found surrounding on the entrance of the nest and the population of this colony was medium in size, of which $>300$ individuals were seen including eggs and broods inside the nest (Fig. 5). Interestingly, it was evident that they were guarding and feeding on fungi growing on the cave wall at the entrance of its nest (white substrate on Fig. 5). This remains to be investigated in more detail to better understand the diet and biology of this diverse ant genus. This is because Carebara are general scavengers and predators, and some have specialized predatory diets, foraging mainly on the ground (Fischer, 2012; Shattuck, 1999). Therefore, fungi might be part of the potential diet of this ant species.

The morphological characters of Carebara panhai sp. n. are suggestive of non-troglomorphic characters. Generally, true cave-adapted characters or troglomorphy of ants are defined by 1) the elongation of appendages, 2) reduction of pigment, 3) reduction to loss of eyes, and 4) loss ability to fly (see discussion in Roncin \& Deharveng, 2003; Naka \& Maruyama, 2018). Carebara panhai sp. n. displays normal morphological forms as presented in other soil species and its related taxa. It clearly exhibits less of a degree than cave-adapted species, only its coloration of minor caste is lighter than other related species of the species group. However, nesting in the twilight zone of cave with colony (workers, eggs and broods) strongly indicates that caves are a proper place for shelter, and therefore this ant is considered here as a cave-dwelling species.

The cave where the new species was discovered developed in a small karstic hill, surrounded by a dense dry evergreen forest. The cave is located at a rather high altitude of 683 meters above sea level. The new species was captured in November 2019, when the temperature of the cave was 22.4 degree Celsius and the relative humidity was $77 \%$ in midday. The temperature significantly decreased to at least 14 degrees Celsius (or lower) at night, which is considered a low temperature in tropical areas. Between November and January, the high altitude of western region of Thailand experiences a short winter when temperatures dramatically drop during the night (can drop to 5 degrees Celsius). Therefore, the presence of Carebara panhai sp. $\mathrm{n}$. in the twilight zone near the entrance of cave might be related to 


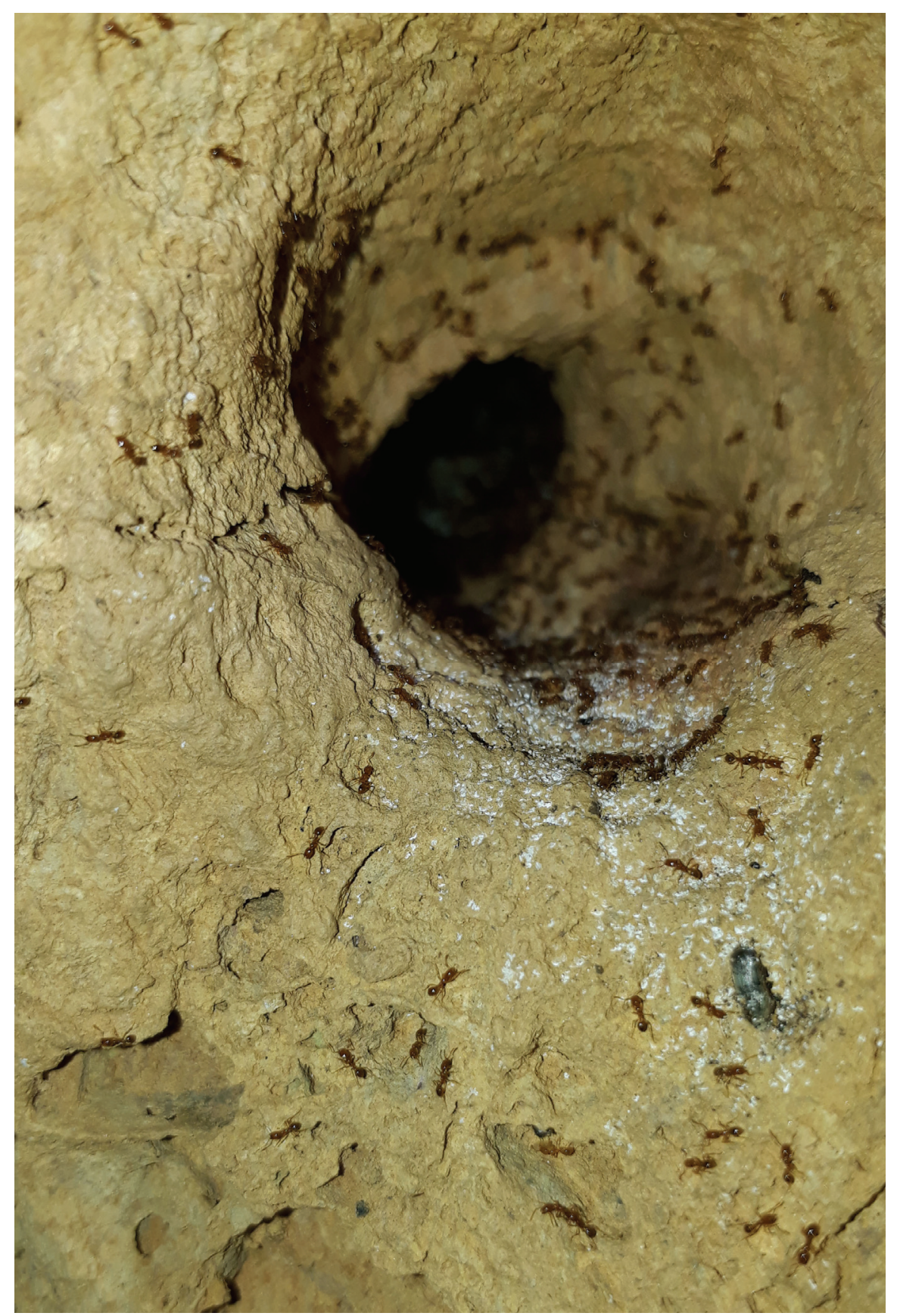

Fig. 5. Nest entrance of Carebara panhai sp. n. on a cave wall. 
the temperature fluctuation or to avoid the hazard environment during this time. We also surveyed and collected soil and surface species of ants in the surrounding areas for years; however, none of the individual of this new species was observed or collected. It seems that its distribution range is probably narrow and might be restricted to the cave or karstic environment. Consequently, the presence of a colony and broods inside the cave strongly indicates that they are a cave-dwelling species. Long-term colonial observation would be advantage for ecological monitoring and habitat preferences of this new species.

To date, 529 species of ants are known from Thailand, and at least 80 species are described as new to science from various types of habitat (Khachonpisitsak et al., 2020). Unfortunately, none of them were described from cave environments. Hence, this new species is the first described species of ant in Thai caves and apparently sheds a new light that caves could provide important localities for the preservation of ant assemblages. We hope that the description of this ant species will encourage other myrmecologists to pay more attention to cave and subterranean habitats.

\section{ACKNOWLEDGMENTS}

This study was supported by the Office of the Higher Education Commission and Centre of Excellence on Biodiversity (project no. BDC-PG2-160009) for the first and the second author and is also supported by The Thailand Research Fund (RSA6280063) for the third author. We thank the Sahakhon Nikhom Community Forest Working Group for partly assisting us in surveying and collecting data in the area.

\section{REFERENCES}

Antwiki. 2020. Carebara. Available from: https://www.antwiki.org/wiki/Carebara (Accessed: 25 November 2020).

Azorsa, F. \& Fisher, B.L. 2018. Taxonomy of the ant genus Carebara Westwood (Formicidae, Myrmicinae) in the Malagasy Region. ZooKeys, 767: 1-149. DOI: https://doi.org/ 10.3897/zookeys.767.21105

Emery, C. 1887. Catalogo delle formiche esistenti nelle collezioni del Museo Civico di Genova. Parte terza. Formiche della regione Indo-Malese e dell'Australia (continuazione e fine). Annali del Museo civico di storia naturale di Genova, 25[=(2)(5)]: 465-473.

Emery, C. 1893. Voyage de M. E. Simon aux îles Philippines (mars et avril 1890). Formicides. Annales de la Société entomologique de France, 62: 259-270.

Emery, C. 1900. Formicidarum species novae vel minus cognitae in collectione Musaei Nationalis Hungarici quas in Nova-Guinea, colonia germanica, collegit L. Biró. Publicatio secunda. Természetrajzi Füzetek, 23: 310-338.

Emery, C. 1924. Hymenoptera. Fam. Formicidae. Subfam. Myrmicinae. [concl.]. Genera Insectorum, 174C: 207-397.

Ettershank, G. 1966. A generic revision of the world Myrmicinae related to Solenopsis and Pheidologeton (Hymenoptera: Formicidae). Australian Journal of Zoology, 14: 73-171.

Fernández, F. 2004. The American species of the myrmicine ant genus Carebara Westwood (Hymentoptera: Formicidae). Caldasia 26(1): 191-238.

Fischer, G. 2012. Ecology, Biogeography and Responses to Habitat Degradation of a Highly Diverse Rainforest Ant Community and Taxonomy of Afrotropical Pheidole Westwood (Hymenoptera, Formicidae). Bonn, Rheinische Friedrich-Wilhelms-Universität Bonn, Dissertation, 228 pp. 
Fischer, G., Azorsa, F. \& Fisher, B.L. 2014. The ant genus Carebara Westwood (Hymenoptera, Formicidae): synonymisation of Pheidologeton Mayr under Carebara, establishment and revision of the C. polita species group. ZooKeys, 438: 57-112.

Forel, A. 1915. Fauna Simalurensis. Hymenoptera Aculeata, Fam. Formicidae. Tijdschrift voor Entomologie, 58: 22-43.

Herwina, H., Mairawita, Yulvita, L., Putri, D., Satria, R., Janra, M.N., Yaherwandi \& Sakamaki, Y. 2020. Ant species composition (Hymenoptera: Formicidae) at a highland agricultural area for wheat and potato in Alahan Panjang, West Sumatera. IOP Conference Series: Earth and Environmental Science, 515: 012018. DOI: https://doi.org/10.1088/ 1755-1315/ 515/1/012018

Khachonpisitsak, S., Yamane, Sk., Sriwichai, P. \& Jaitrong, W. 2020. An updated checklist of the ants of Thailand (Hymenoptera, Formicidae). ZooKeys, 998: 1-182. DOI: https:// doi.org/10.3897/zookeys.998.54902

Naka, T. \& Maruyama, M. 2018. Aphaenogaster gamagumayaa sp. nov.: the first troglobiotic ant from Japan (Hymenoptera: Formicidae: Myrmicinae). Zootaxa, 4450(1): 135-141. DOI: https://doi.org/10.11646/zootaxa.4450.1.10

Pfeiffer, M., Mezger, D., Hosoishi, S., Yahya, B.E. \& Kohout, R.J. 2011. The Formicidae of Borneo (Insecta: Hymenoptera): a preliminary species list. Asian Myrmecologist, 4: 958.

Roncin, E. \& Deherveng, L. 2003. Leptogenys khammouanensis sp. nov. (Hymenoptera: Formicidae). A possible troglobitic species of Laos, with a discussion on cave ants. Zoological science, 20(7): 919-924.

Shattuck, S.O. 1999. Australian Ants: Their Biology and Identification. CSIRO Publishing, Collingwood, VIC. 226 pp.

Wheeler, W.M. 1909. Ants of Formosa and the Philippines. Bulletin of the American Museum of Natural History, 26: 333-345. 